\title{
Analysis of the Systematic Errors Found in the Kipp \& Zonen Large-Aperture Scintillometer
}

\author{
B. Van Kesteren • O. K. Hartogensis
}

Received: 24 February 2010 / Accepted: 12 November 2010 / Published online: 3 December 2010

(C) The Author(s) 2010. This article is published with open access at Springerlink.com

\begin{abstract}
Studies have shown a systematic error in the Kipp \& Zonen large-aperture scintillometer (K\&ZLAS) measurements of the sensible heat flux, $H$. We improved on these studies and compared four K\&ZLASs with a Wageningen large-aperture scintillometer at the Chilbolton Observatory. The scintillometers were installed such that their footprints were the same and independent flux measurements were made along the measurement path. This allowed us to compare $H$ and the direct scintillometer output, the refractive index structure parameter, $C_{n}^{2}$. Furthermore, spectral analysis was performed on the raw scintillometer signal to investigate the characteristics of the error. Firstly, correlation coefficients $\geq 0.99$ confirm the robustness of the scintillometer method, and secondly we discovered two systematic errors: the low- $C_{n}^{2}$ error and the high- $C_{n}^{2}$ error. The low- $C_{n}^{2}$ error is a non-linear error that is caused by high-frequency noise, and we suspect the error to be caused by the calibration circuit in the receiver. It varies between each K\&ZLAS, is significant for $H \leq 50 \mathrm{~W} \mathrm{~m}^{-2}$, and we propose a solution to remove this error using the demodulated signal. The high- $C_{n}^{2}$ error identified by us is the systematic error found in previous studies. We suspect this error to be caused by poor focal alignment of the receiver detector and the transmitter light-emitting diode that causes ineffective use of the Fresnel lens in the current Kipp \& Zonen design. It varies between each K\&ZLAS (35\% up to $240 \%$ ) and can only be removed by comparing with a reference scintillometer in the field.
\end{abstract}

Keywords $C_{n}^{2} \cdot$ Eddy covariance $\cdot$ Instrument intercomparison · Large-aperture scintillometer $\cdot$ Scintillation $\cdot$ Sensible heat flux

B. Van Kesteren $(\bowtie) \cdot$ O. K. Hartogensis

Meteorology and Air Quality Group, Wageningen University, P.O. Box 47, 6700 AA, Wageningen

The Netherlands

e-mail: bram.vankesteren@wur.nl 


\section{Introduction}

Many applications in meteorology and hydrology rely on accurate estimates of the area-averaged sensible heat flux, $H$. The large-aperture scintillometer (LAS) yields this property from area-averaged measurements of the refractive index structure parameter, $C_{n}^{2}$. Initially, only prototype large-aperture scintillometers were employed and research focussed on theoretical and practical issues regarding the method (Ochs and Wang 1978; Wang et al. 1978; De Bruin et al. 1995; Nieveen et al. 1998; Meijninger and De Bruin 2000; Meijninger 2003). Based on this research the large-aperture scintillometer was made commercially available and has been widely used since (Asanuma and Iemoto 2007; Hartogensis 2007; Kleissl et al. 2008). This inspired two studies to investigate the instrument variability of two commercially available large-aperture scintillometers, namely the Kipp \& Zonen large-aperture scintillometer (Kipp \& Zonen, Delft, the Netherlands) and the boundary-layer scintillometer (Scintec, Rottenburg, Germany) (Kleissl et al. 2008, 2009).

In the first study Kleissl et al. (2008) conducted two field experiments with six Kipp \& Zonen large-aperture scintillometers using horizontal and slant paths. During the first experiment the instruments stood on two slopes that overlook relatively flat grassland. The scintillometer path was horizontal, had a length of $2 \mathrm{~km}$ and an average effective height, $z_{\text {eff }}$, of $43 \mathrm{~m}$. The distance between the outermost scintillometers was $54 \mathrm{~m}$ at one side and $26 \mathrm{~m}$ at the other. An eddy-covariance system installed at a height of $2.93 \mathrm{~m}, 1.3 \mathrm{~km}$ south of the transect served as an independent reference for $H$. During the second experiment over arid shrubland the transmitters were located on an $80 \mathrm{~m}$ high ridge and the receivers almost at surface level in the plains below. The scintillometer path was slanted, had a length of $1.3-2.8 \mathrm{~km}$, and $z_{\text {eff }}$ varied between 15 and $24 \mathrm{~m}$. The lateral distance between any two receivers was $30 \mathrm{~m}$. An eddy-covariance system installed at a height of $2.85 \mathrm{~m}$ at the centre of the scintillometer path served as an independent reference for $H$.

In comparing the scintillometers with each other, Kleissl et al. (2008) chose to evaluate the more common variable $H$ rather then a rescaled $C_{n}^{2}$ to account for differences in $z_{e f f}$ between the scintillometers. They showed that the scintillometers correlate very well with each other $(r>0.98)$, which is superior to that reported for eddy-covariance flux intercomparisons (Kleissl et al. 2008). However, the magnitude of $H$ varied considerably between the different sensors. Overall $H_{L A S}$ overestimated $H_{E C}$ by $2-17 \%$ and regression-slope differences between the instruments were typically $6 \%$ with a maximum of up to $21 \%$. One of the scintillometers was sent back to Kipp \& Zonen to repair a photodiode detector that was out of focus. Kleissl et al. (2008) observed that $H$ from this scintillometer was on average 18\% larger than that of another scintillometer before the repair and on average $18 \%$ smaller than that of the same scintillometer after the repair. They therefore considered uncertainties in the effective aperture size, which is related to the detector alignment, as the prime suspect for the observed differences in regression slope (Kleissl et al. 2008).

In the second study Kleissl et al. (2009) extended and improved on the first study. Firstly, they made improvements by making the scintillometer footprints closer and better defined through measurements along a path of $635 \mathrm{~m}$ over a homogeneous peanut field. The measurement height varied between 1.50 and $1.66 \mathrm{~m}$ above ground level and the lateral distances between 0.62 and $2.19 \mathrm{~m}$ at one side and $4.5 \mathrm{~m}$ on the other side. Secondly they extended by comparing two types of large-aperture scintillometers instead of one, namely one Kipp $\&$ Zonen large-aperture scintillometer (K\&ZLAS) with three boundary layer scintillometers (BLSs). In this way they could determine whether the differences in the regression slope that were observed in the previous study are specific to the K\&ZLAS or typical of other types as well. Unfortunately independent flux measurements for comparison were not available in 
this experiment. Kleissl et al. (2009) again compared $H$ and found correlation coefficients were greater than 0.97 for all scintillometer comparisons and a BLS inter-instrument variability of the regression slope less than $3 \%$. The difference, however, in the regression slope with the K\&ZLAS was more than $20 \%$. This suggested that, indeed, the observed differences are due to problems in the K\&ZLAS (Kleissl et al. 2009).

The goal of the present study is to perform a more detailed and systematic analysis of the differences that were observed by Kleissl et al. (2008, 2009). To achieve this we conducted an experiment under more controlled conditions, compared the scintillometer fluxes with independent flux measurements, and did a spectral analysis of the raw scintillometer signal. From August 2007 until November 2008 we deployed four Kipp \& Zonen large-aperture scintillometers and one Wageningen large-aperture scintillometer at the Chilbolton Observatory, UK. Unlike Kleissl et al. (2008, 2009) we installed all transmitters and receivers at exactly the same height of $4.37 \mathrm{~m}$ above ground level. We could therefore compare the more direct scintillometer output, $C_{n}^{2}$, rather than $H$, since we did not have to consider the height dependency of $C_{n}^{2}$. A vibration free installation was ensured by locating the scintillometers on the special benches available in the research cabins. At approximately the same height as the scintillometers (4.46 m above ground level) we placed an eddy-covariance system halfway along the scintillometer paths to measure $H$ as an independent reference. In addition to the more controlled set-up our experiment has the advantage over the two Kleissl studies (2008, 2009) in that we sampled the raw scintillometer signal with a $500 \mathrm{~Hz}$ sampling frequency. This sampling frequency allowed us to do spectral data analysis and so investigate the reasons behind the systematic differences and propose, at least partly, solutions for them.

\section{Theory}

A large-aperture scintillometer consists of a transmitter that emits a beam of electromagnetic radiation at near-infrared wavelength and a receiver that registers the beam intensity, $I$, over distances of typically 500-5,000 m. On the way to the receiver the emitted electromagnetic wave is diffracted by turbulent eddies. These eddies all have different air densities and thus different refractive indices, $n$. Since eddies move, the refractive-index field continuously changes, causing the measured intensity to fluctuate. If the aperture of the scintillometer is much smaller than the outer scale of turbulence, yet much larger than the inner scale of turbulence the intensity fluctuations can be directly related to the refractive-index structure parameter, $C_{n}^{2}$ (Wang et al. 1978):

$$
C_{n}^{2}=1.12 \sigma_{\ln I}^{2} D^{7 / 3} L^{-3}
$$

where $\sigma_{\ln I}^{2}$ is the variance of the logarithmic intensity, $D$ is the aperture size, and $L$ is the path length.

The procedure to estimate the sensible heat flux, $H$, from $C_{n}^{2}$ consists of two steps and needs additional standard measurements of pressure, temperature, humidity, and wind speed. The first step is to derive the structure parameter of temperature, $C_{T}^{2}$, from $C_{n}^{2}$, and we use Eq. 12 from Moene (2003) and the values of $A_{t}$ and $A_{q}$ as given in Andreas $(1988,1989)$. The second step is to iteratively solve $H$ from $C_{T}^{2}$ using Monin-Obukhov similarity theory (MOST). In our study the similarity relations of Andreas (1988) are used with the constants $c_{1}=4.9$ and $c_{2}=6.1$ (Moene et al. 2004). The friction velocity $u_{*}$ is obtained from the standard MOST flux-profile relationship (see Eqs. 12-15 in De Bruin et al. (1995)). The wind speed was measured at $10 \mathrm{~m}$ above the ground and the roughness length $z_{0}$ was estimated 
as $0.01 \mathrm{~m}$. A more elaborate description of the flux calculations can be found in Meijninger et al. (2002), Moene et al. (2004), and Van Kesteren (2008).

\section{Experiment and Instrumentation}

This section describes the set-up of the field experiment that consisted of two parts: the first, most comprehensive, part (Experiment 1) took place from 23 July until 10 September 2007. The second part (Experiment 2) took place from 17 October until 19 November 2008.

\subsection{Description of the Field Site}

The experiment was performed at the Chilbolton Observatory, UK $\left(51.1445^{\circ} \mathrm{N}\right.$ and $1.437^{\circ} \mathrm{W}$ ), which is operated by the Radio Communications Research Unit (RCRU) of the Space Science and Technology Department at the Rutherford Appleton Laboratory (RAL). Measurements were made at their so-called test range, a nearly horizontal strip of grassland (grass $<0.1 \mathrm{~m}$ tall), approximately $15 \mathrm{~m}$ wide and $500 \mathrm{~m}$ long with identical research cabins at each end (see Fig. 1).

The research cabins were equipped with benches that stood independent of the cabins on large concrete pillars anchored deep into the ground. The benches provided the necessary space to place all the scintillometers at the same height $(4.37 \mathrm{~m})$. Consequently we could ignore the height dependency of $C_{n}^{2}$ and directly compare the instruments without any additional measurements. Furthermore the benches ensured a vibration-free installation so that intensity fluctuations induced by vibrations of the instrument itself did not disturb the measurements.

To account for the small height differences in the topography along the test range we determined the effective height of the scintillometers following Hartogensis et al. (2003). In addition we used their Eq. 15 to give a stability independent effective height formulation, which is appropriate for this case. The topographical data are obtained from a survey carried out in 1980 by J.A. Storey and Partners. The topographic levels of the area around the test range were sampled at 50-m intervals. The resulting effective height is $3.90 \mathrm{~m}$.

At the east side of the range an eddy-covariance mast was placed halfway between the cabins at approximately the same height $(4.46 \mathrm{~m})$ as the scintillometers to obtain independent flux measurements. The height, together with the relatively short scintillometer path length
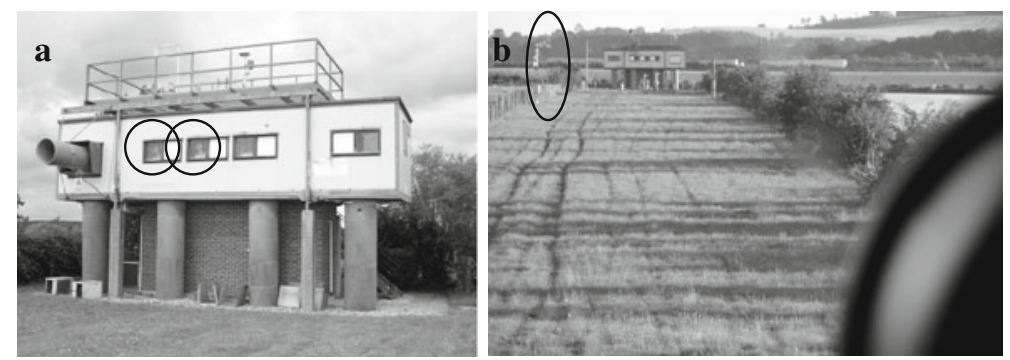

Fig. 1 a K\&ZLAS receiver cabin - the scintillometers are visible behind the two encircled windows. b View from the K\&ZLAS transmitter to the receiver cabin-the eddy-covariance tower is encircled 
Table 1 Overview of the large-aperture scintillometers (LAS) by abbreviation, type, serial number (S/N), owner, deployment time, and experiment number (Exp)

\begin{tabular}{llllll}
\hline LAS & Type & S/N & Owner & Deployment (dd/mm/year) & Exp. \\
\hline WagLAS & Wageningen LAS & 99004 & MAQ & $08 / 08 / 2007-10 / 09 / 2007$ & 1 \\
K\&ZLAS09 & Kipp \& Zonen LAS & 040009 & CEH & $08 / 08 / 2007-19 / 11 / 2008$ & $1+2$ \\
K\&ZLAS01 & Kipp \& Zonen LAS & 030001 & CEH & $17 / 10 / 2008-21 / 10 / 2008$ & 2 \\
K\&ZLAS30 & Kipp \& Zonen LAS & 060030 & King's College & $21 / 10 / 2008-03 / 11 / 2008$ & 2 \\
K\&ZLAS29 & Kipp \& Zonen LAS & 060029 & King's College & $03 / 11 / 2008-19 / 11 / 2008$ & 2 \\
\hline
\end{tabular}

of $500 \mathrm{~m}$, ensured similar footprints for the scintillometers and the eddy-covariance system. Consequently we could compare the fluxes estimated by the two methods.

The standard additional meteorological measurements were made on site and obtained from the British Atmospheric Data Centre (Wrench 2003-2010).

\subsection{Specification of the Scintillometers}

Two large-aperture scintillometer types were used in our study: the first, a prototype built by the Meteorology and Air Quality group of Wageningen University, is based on the work of Ochs and Wang at NOAA and called the Wageningen large-aperture scintillometer (WagLAS) (Ochs and Wang 1978; Wang et al. 1978; De Bruin et al. 1995). The second is a commercial large-aperture scintillometer from Kipp \& Zonen (K\&ZLAS) that has been developed based on the WagLAS (Meijninger 2003).

There are several differences between the WagLAS and the K\&ZLAS. First of all, the K\&ZLAS uses Fresnel lenses and the WagLAS spherical concave mirrors to collimate the beam and focus it onto the detector. Secondly, in the K\&ZLAS the transmitting light-emitting diode and receiving detector are connected to the housing with only limited adjusting possibilities, whereas the light-emitting diode and detector in the WagLAS are mounted on high-precision positioning devices. Thirdly, the K\&ZLAS has an additional circuit on the board of the receiver electronics to calibrate the signal (Meijninger 2003), which the WagLAS does not have. Less important differences are the wavelength used $(0.88 \mu \mathrm{m}$ for the K\&ZLAS versus $0.94 \mu \mathrm{m}$ for the WagLAS) and the shape of their housing (round for the KippLAS versus square for the WagLAS).

In total five large-aperture scintillometers from the Meteorology and Air Quality group (MAQ), the Centre for Ecology and Hydrology (CEH) from Wallingford, UK, and King's College London, UK, were deployed in this study (Table 1). The K\&ZLAS09 was the only scintillometer present during both Experiments 1 and 2.

\subsection{Design of the Experiment}

During Experiment 1 the scintillometers were separated $1.5 \mathrm{~m}$ and their beams crossed to match their footprints as much as possible. To prevent crosstalk the transmitters were directed in opposite direction (see Fig. 2). A CSAT3 sonic anemometer (Campbell Sci., Logan, USA) and a LiCor-7500 fast response $\mathrm{H}_{2} \mathrm{O}-\mathrm{CO}_{2}$ sensor (LiCor, Lincoln, USA) placed directly below it were installed on the eddy-covariance mast. A CR23X data logger (Campbell Sci., UK) recorded raw $20-\mathrm{Hz}$ data. The fluxes from the eddy-covariance system were determined 


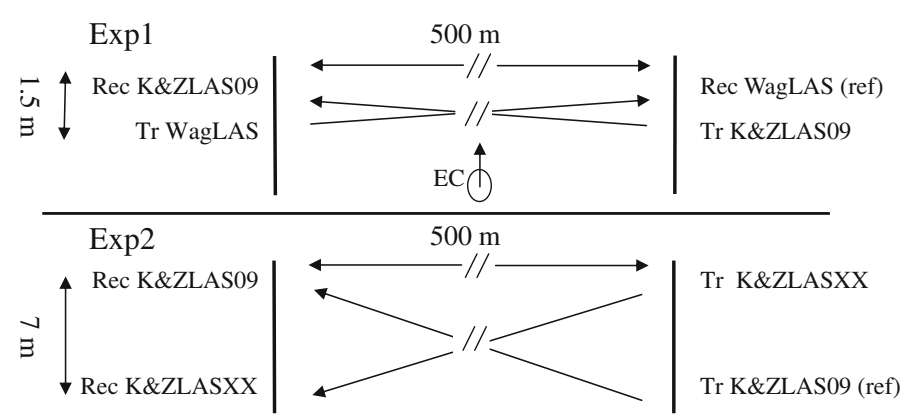

Fig. 2 Experimental set-up at the Chilbolton Observatory during Experiments 1 and 2

using the processing package (ECpack) from Wageningen University (Van Dijk et al. 2004). The following corrections and rotations were applied: the Schotanus correction, linear detrending of the data, the planar-fit rotation, and the frequency response correction (Schotanus et al. 1983; Moore 1986; Kaimal and Finnigan 1994).

Experiment 2 had a different set-up to Experiment 1. The scintillometers were now separated by $7 \mathrm{~m}$ and were directed in the same direction with their beams crossing. Despite the fact that this separation distance exceeded the beam width of $5 \mathrm{~m}(\approx 1 \%$ of the path length) we verified that the receivers only observed their corresponding transmitter. Verification was made by switching off one transmitter at a time; when its corresponding receiver signal fell to zero, the separation distance was confirmed as large enough. Furthermore the set-up was different because the eddy-covariance system was no longer present and the data-logger settings were changed (see following section). Otherwise everything remained the same.

\subsection{Logging and Filtering of the Scintillometer Data}

The large-aperture scintillometers are designed to provide two signal outputs both of which were recorded. The first signal is the received signal intensity, $I$, the so-called demodulated signal, which is related to $C_{n}^{2}$ as shown in Eq. 1. During our experiment we recorded the demodulated-signal variance, but for use in Eq. 1 the variance first had to be converted to a lognormal variance. Furthermore we logged 1-min variances from which we generated longer-term (30-min) variances for comparison of scintillometer data with eddy-covariance data.

Generating $N$-min variances from $m$-min variances uses (Oncley 2007)

$$
\left(\sigma_{I}^{2}\right)^{N}={\overline{I^{\prime} I^{\prime}}}^{N}=\frac{1}{m} \sum_{j=1}^{m}\left({\overline{I^{\prime} I^{\prime}}}^{j}+\bar{I}^{j} \bar{I}^{j}\right)-\frac{1}{m} \sum_{j=1}^{m} \bar{I}^{j} \frac{1}{m} \sum_{j=1}^{m} \bar{I}^{j},
$$

where $\left(\sigma_{I}^{2}\right)^{N}$ is the variance of $I$ over the time interval $N$. That is, the squared mean over the sub-intervals is subtracted from the mean of the sums of the sub-interval variances and the squared sub-interval means. Transforming from normal distributed variance to log-normal distributed variance is done using

$$
\sigma_{\ln I}^{2}=\ln \left(1+\frac{\sigma_{I}^{2}}{\bar{I}^{2}}\right)
$$


which is valid only when the fluctuations are small compared to the mean value, as is the case for the intensity fluctuations.

The second signal that is recorded from the large-aperture scintillometer is the $V_{C_{n}^{2}}$ signal, which is related to $C_{n}^{2}$ by

$$
C_{n}^{2}=10^{-12+V_{C_{n}}}
$$

where $V_{C_{n}^{2}}$ is the signal obtained from the demodulated signal after being processed by the receiver analogue electronics following Eq. 1. It is important to note that part of this analogue processing is to filter the demodulated signal with a band-pass filter between 0.01 and $400 \mathrm{~Hz}$. In this filter the lower limit is set to exclude absorption fluctuations of $I$ (Nieveen et al. 1998), whereas the upper limit is set to exclude electronics noise.

In Experiment 1 two CR9000 data loggers (Campbell Sci., Inc.) differentially sampled and stored the demodulated signal with $500 \mathrm{~Hz}$ sampling frequency and an integration time of $40 \mu \mathrm{s}$. Also a CR23X and a CR10 (both Campbell Sci., Inc.) differentially sampled the demodulated signal and $V_{C_{n}^{2}}$ signal with $1 \mathrm{~Hz}$ sampling frequency and an integration time of $250 \mu$ s. Subsequently the CR23X and CR10 stored the 1-min mean and standard deviation of these two signals. Thus for Experiment $1 C_{n}^{2}$ can be calculated from the demodulated raw signal as well as from the statistics of both scintillometer signals. In Experiment 2 a CR9000 data logger differentially sampled and stored the demodulated signal with a $500 \mathrm{~Hz}$ sampling frequency and an integration time of $40 \mu \mathrm{s}$. Also the CR9000 differentially sampled the demodulated signal and $V_{C_{n}^{2}}$ signal with $500 \mathrm{~Hz}$ sampling frequency and an integration time of $40 \mu \mathrm{s}$. Subsequently the CR9000 stored the 1-min mean and standard deviation of these two signals. Thus for Experiment $2 C_{n}^{2}$ can be calculated from the demodulated raw signal as well as from the statistics of both scintillometer signals.

To check on and improve data quality in Experiment 1 we applied data filters. Data were excluded when the signal variance was not solely determined by refractive index fluctuations, which happened during rain, fog, and dust/smoke events. Furthermore the flux comparison was limited to unstable daytime data. The exact data filters for each variable are described in Van Kesteren (2008).

In Experiment 2 data filters were different. We only excluded data when the mean modulated signal was too low (indicating fog) or when it rained.

\section{Results}

\subsection{Comparison of $H_{E C}$ with $H_{W a g L A S}$ and $H_{K \& Z L A S 09}$}

Firstly, we compare the WagLAS and the K\&ZLAS09 with the independent eddy-covariance (EC) system, and to this end we present in Fig. $3 H_{W a g L A S}$ and $H_{K \& Z L A S 09}$ (derived from the $V_{C_{n}^{2}}$ signal) plotted against $H_{E C}$. Figure 3 shows that the scatter is small, resulting in high correlation coefficients, $r$, of 0.97 and 0.96 respectively. However, both scintillometers estimate a greater $H$ than the eddy-covariance method. The regression fit of $H_{W a g L A S}$ has an offset of $4.8 \mathrm{~W} \mathrm{~m}^{-2}$ and the slope is 1.07 and that of $H_{K \& Z L A S 09}$ has an offset of $21 \mathrm{~W} \mathrm{~m}^{-2}$ and the slope is 1.37 .

The most striking characteristic from these regression data is the difference in regression slope between the two scintillometers. As the effective height of both scintillometers and their footprints are identical we expect their regression slopes to be similar. Instead, $H_{K \& Z L A S 09}$ is $28 \%$ greater than $H_{W a g L A S}$ and $37 \%$ greater than $H_{E C}$. Furthermore Fig. 3 shows that 

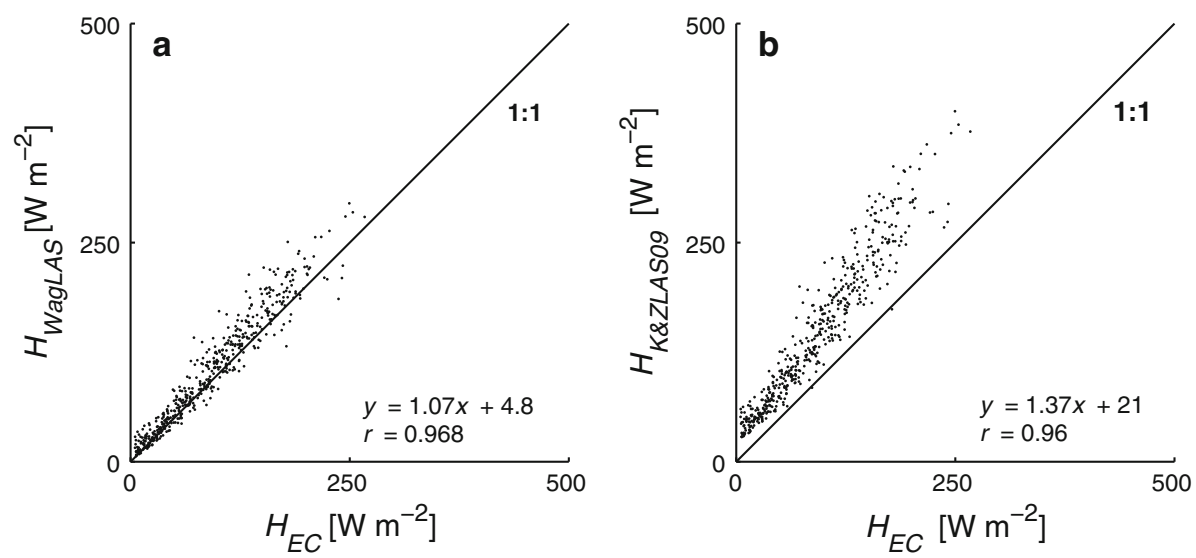

Fig. 3 a Comparison of the sensible heat flux from the Wageningen LAS with eddy covariance, and $\mathbf{b}$ the sensible heat flux from the Kipp \& Zonen LAS with eddy covariance. Each data point is derived from 30-min statistics

the relative difference between $H_{K \& Z L A S 09}$ and $H_{E C}$ is dependent on the magnitude of the flux. There is a different regression slope for $H_{E C}<50 \mathrm{~W} \mathrm{~m}^{-2}$ than for $H_{E C}>50 \mathrm{~W} \mathrm{~m}^{-2}$, whereas for $H_{W a g L A S}$ no such difference in the regression slope can be observed. This is even clearer when plotted on a logarithmic scale (not shown).

Figure 3, thus, corroborates the performance of the WagLAS and K\&ZLAS09 found in the literature: the WagLAS has been shown to give accurate flux estimates (De Bruin et al. 1995; Green 2001; Beyrich et al. 2002; Meijninger et al. 2002, 2006), whereas the K\&ZLAS has been shown to have systematic errors of up to $20 \%$ (Kleissl et al. 2008, 2009). In the following we therefore use the WagLAS as the reference scintillometer.

\subsection{Comparison $C_{n}^{2}$ Between WagLAS and K\&ZLASs}

To investigate the systematic differences between the scintillometers we will compare the scintillometers directly with each other by looking at $C_{n}^{2}$ instead of $H$. For consistency, for $C_{n}^{2}$ derived from the $V_{C_{n}^{2}}$ signal, we use the same collection of data points as in Fig. 3. As can be seen in Fig. 4 the correlation between the scintillometers is 0.997 , a high correlation that underlines that indeed the differences are systematic and not random. Furthermore, it can be seen that the K\&ZLAS09 overestimates $C_{n}^{2}$ in two ways. Firstly, Fig. 4a shows a linear overestimation affecting high values of $C_{n}^{2}$. It is this overestimation that leads to the large regression slope that can be observed in Fig. $3 b$, and from here on we refer to this overestimation as the high- $C_{n}^{2}$ error. Secondly, Fig. $4 \mathrm{~b}$ shows a non-linear overestimation affecting low values of $C_{n}^{2}$. It is this overestimation that leads to the bending that can be observed in Fig. $3 b$, and from here on we refer to this overestimation as the low- $C_{n}^{2}$ error.

To gain insight into what time scales contribute to the variance of the demodulated, raw, signal and thus to $C_{n}^{2}$ we present typical frequency spectra in Fig. 5. Figure 5a shows the spectrum for a strong turbulence case (high $C_{n}^{2}$ ) and Fig. $5 \mathrm{~b}$ shows the spectrum for a weak turbulence case (low $C_{n}^{2}$ ). We scaled the spectra such that the area below the curves is proportional to the variance and that the axes of both spectra are the same.

Figure 5a illustrates the linear high- $C_{n}^{2}$ error. The frequencies lower than $100 \mathrm{~Hz}$ represent signal-intensity fluctuations due to refractive-index fluctuations, i.e. scintillations. It is in this 

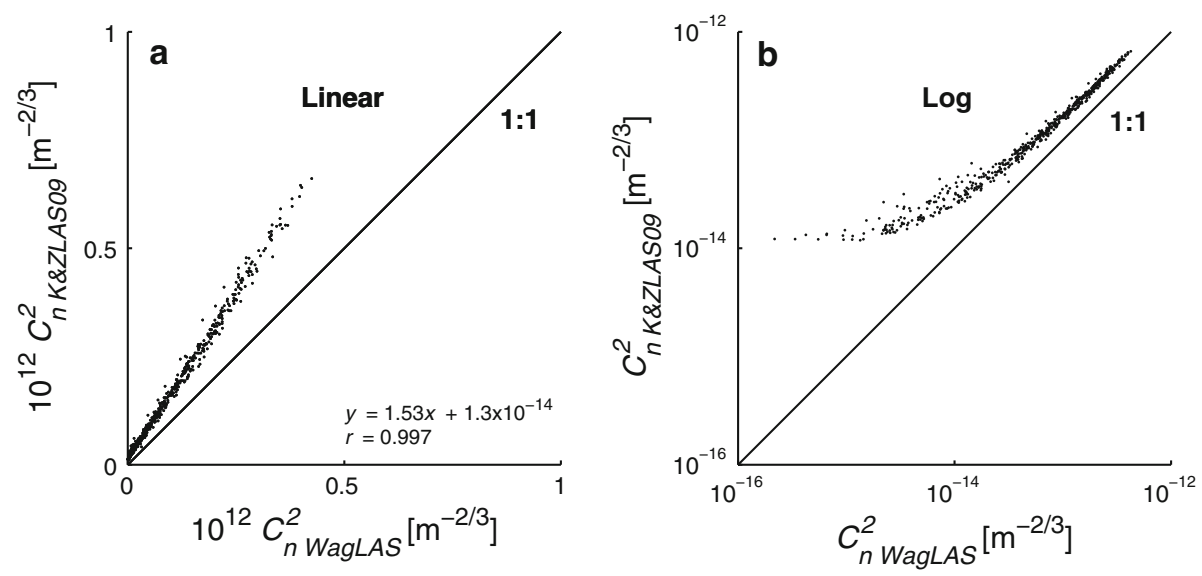

Fig. 4 Comparison of $C_{n}^{2}$ from the Wageningen LAS with Kipp \&Zonen LAS on a linear scale and $\mathbf{b}$ a logarithmic scale. Each data point is derived from 30-min statistics
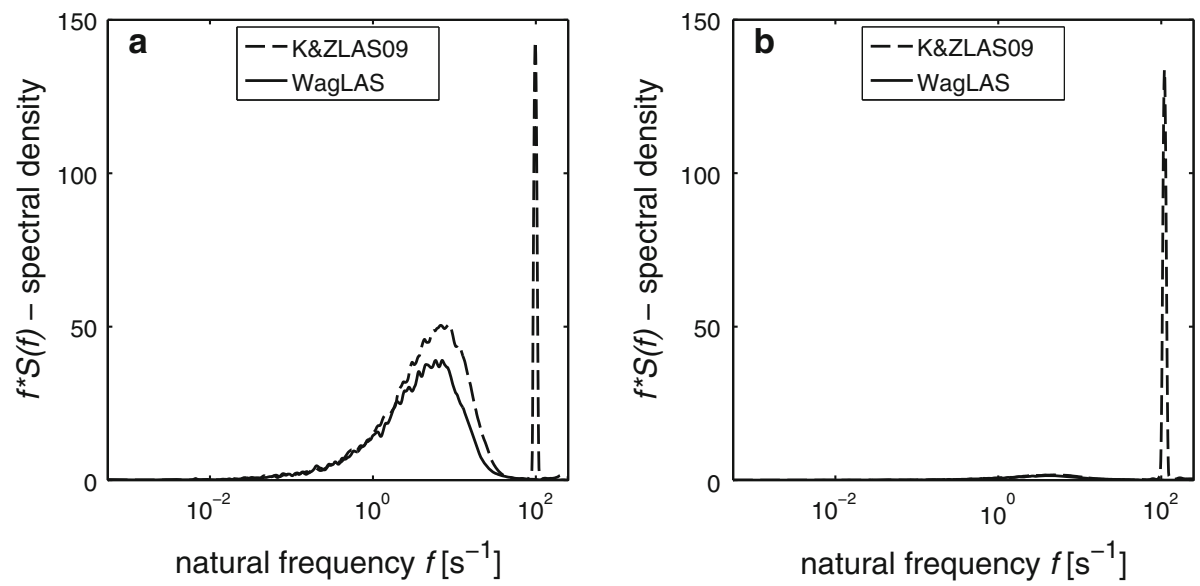

Fig. 5 Frequency spectra of the Kipp \& Zonen LAS (S/N 040009) and the Wageningen LAS demodulated signal, plotted such that the area below the curve is proportional to the variance. Spectra are for 26 August 2007 for the interval a 1300-1330 UTC and b 1730-1800 UTC

region that the K\&ZLAS09 spectral density is greater than the WagLAS spectral density. At about $100 \mathrm{~Hz}$ a distinct peak contaminates the frequency spectrum. For strong turbulence the peak has a negligible contribution to the overall signal-intensity variance and thus to $C_{n}^{2}$. Consequently, for strong turbulence the K\&ZLAS09 $C_{n}^{2}$ overestimation is dominated by its elevated spectral density at frequencies smaller than $100 \mathrm{~Hz}$. Apparently the K\&ZLAS09 elevated spectral density has a fixed ratio with the WagLAS spectral density given the linear relationship between their resulting $C_{n}^{2}$ values as observed in Fig. 4a.

Figure $5 \mathrm{~b}$ illustrates the non-linear low- $C_{n}^{2}$ error. For this weak-turbulent case the spectral densities caused by scintillations are considerably lower than those for the strong-turbulent case. Although not distinguishable with the applied axis scaling the K\&ZLAS09 spectral density caused by scintillations is still systemically greater than that of the WagLAS. However, at $100 \mathrm{~Hz}$, the non-scintillation peak is still present with roughly the same magnitude. 

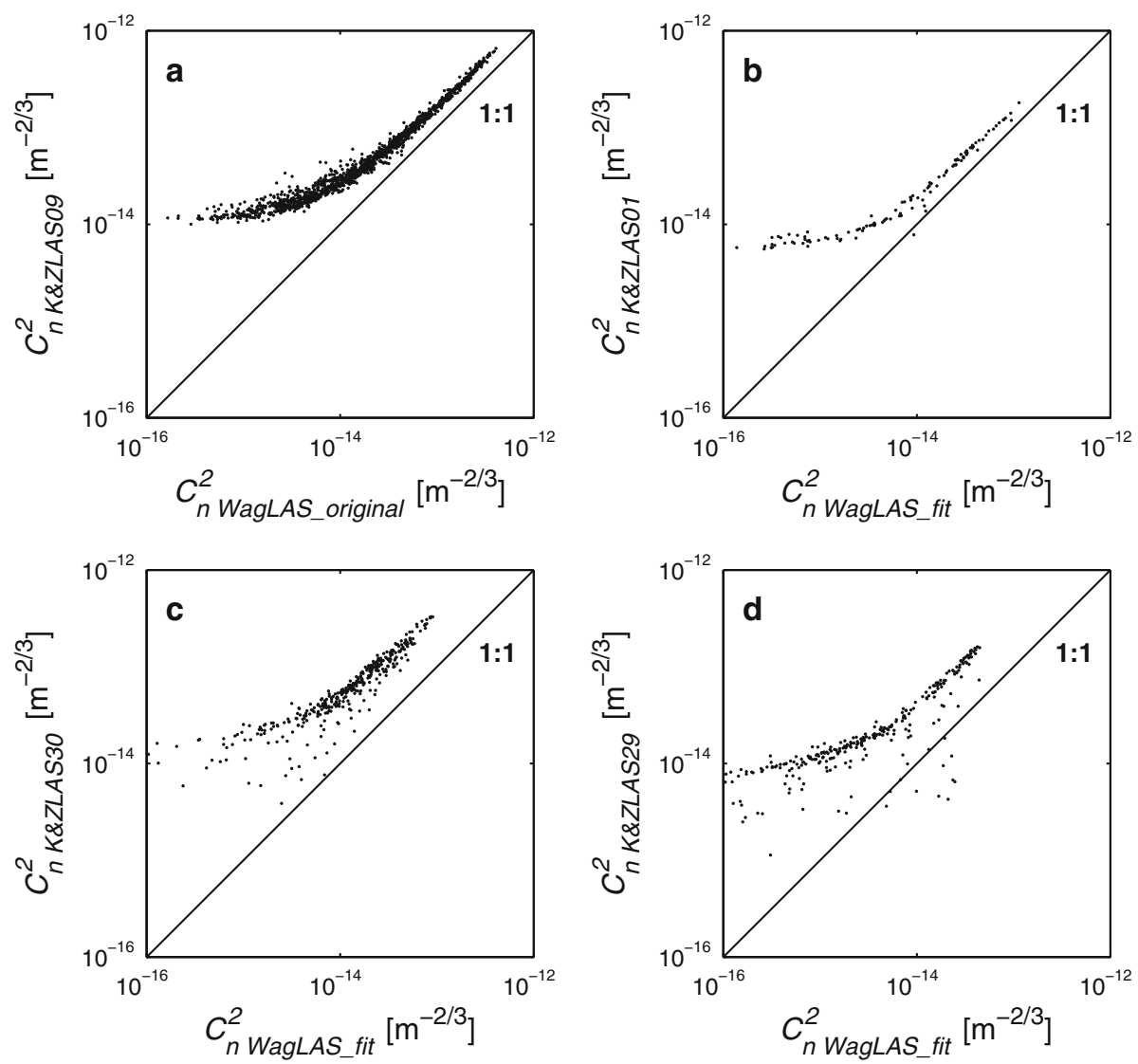

Fig. $6 C_{n}^{2}$ comparison of K\&ZLAS09 against a the reference WagLAS, b K\&ZLAS01, $\mathbf{c}$ K\&ZLAS30, d K\&ZLAS29. See text for further explanation on the difference between $C_{n W a g L A S \_o r i g i n a l}^{2}$ and $C_{n \text { WagLAS_fit }}^{2}$. Each data point is derived from 30-min statistics

Since the magnitude of the peak remains the same independent of the turbulent intensity the contribution of the peak to the overall signal-intensity variance becomes less significant with increasing turbulent intensity. This explains the non-linear behaviour for low $C_{n}^{2}$ observed in Fig. 4b.

To show that the two systematic errors incurred by the K\&ZLAS09 are not an exception we introduce Fig. 6, which combines results of Experiment 1 and 2 for comparing the WagLAS with the K\&ZLASs. Note that in Experiment 2 the WagLAS was not available and the K\&ZLAS09 was the only scintillometer available during both Experiments 1 and 2 . We therefore fitted a curve to the WagLAS-K\&ZLAS09 comparison and applied that to the K\&ZLAS09 measurements during Experiment 2. This explains the terms $C_{n \text { WagLAS_original }}^{2}$ in Fig. 6a and $C_{n W a g L A S_{-} \text {fit }}^{2}$ in Fig. 6b-d. As in Fig. $4 C_{n}^{2}$ was calculated from the $V_{C_{n}^{2}}$ signal.

The main conclusion that can be drawn from Fig. 6 is that indeed all four K\&ZLAS instruments have the systematic high- and low- $C_{n}^{2}$ errors. In addition, it can be seen that the magnitude of the high- and low- $C_{n}^{2}$ errors differs for each scintillometer. 


\subsection{Discussion and Solutions of the K\&ZLAS Systematic Errors}

In the previous section we showed that the systematic error in the K\&ZLAS09 is present in all K\&ZLASs, that it is different for each K\&ZLAS, and that it consists of two parts: the low- $C_{n}^{2}$ error and the high- $C_{n}^{2}$ error. These two parts we more closely consider in the next. In Sect. 4.3.1 we deal with the low- $C_{n}^{2}$ error and subsequently with the high- $C_{n}^{2}$ error in Sect. 4.3.2.

\subsubsection{Discussion and Solution of the Low- $C_{n}^{2}$ Error}

The low- $C_{n}^{2}$ error was already noted on site during Experiment 1, and for that reason we did three tests to investigate the source of this error. Firstly, we connected the direct-current power supply to batteries to exclude possible interference from the mains power supply. Secondly, we turned off the WagLAS, switched the K\&ZLAS transmitter and receiver, and sampled with the WagLAS CR9000. Thus we could check whether external interference in the K\&ZLAS09 receiver cabin or measurements in the K\&ZLAS09 CR9000 were the source of errors. Lastly, we used the WagLAS transmitter instead of the K\&ZLAS09 transmitter to check whether the transmitter was the source. In all spectra created from these test data the peak amplitude and position remained unchanged. Consequently we concluded that the low- $C_{n}^{2}$ error was generated by an internal source within the K\&ZLAS09 receiver.

To investigate what source in the receiver causes the low- $C_{n}^{2}$ error, we consider the different output signals of the scintillometer, see Sect. 3.4. In Sect. 4.2 we implicitly neglected any difference between the two signals and linked the low- $C_{n}^{2}$ error observed in Fig. 4 with the spectral peak observed in Fig. 5. However, to learn about the source of the low- $C_{n}^{2}$ error we consider below in more detail the $C_{n}^{2}$ estimates from the $V_{C_{n}^{2}}$ signal and the demodulated signal. Furthermore we look at the data logger's influence on these signals by considering the logger's signal integration times.

Figure 7 depicts the 30-min $C_{n}^{2}$ comparison between the K\&ZLAS09 and WagLAS, where $C_{n}^{2}$ is evaluated in three different ways. Firstly, $C_{n}^{2}$ is derived from the $V_{C_{n}^{2}}$ signal sampled on the CR23X with a frequency of $1 \mathrm{~Hz}$ and an integration time of $250 \mu \mathrm{s}$. Most users evaluate $C_{n}^{2}$ in this way. Secondly, $C_{n}^{2}$ is derived from the demodulated signal sampled on the CR23X with a frequency of $1 \mathrm{~Hz}$ and an integration time of $250 \mu \mathrm{s}$. The 1-min statistics from this signal are averaged and transformed to $C_{n}^{2}$ using Eqs. 2, 3, and 1. Since Kipp \&

Fig. 7 Comparison of 30-min averaged $C_{n}^{2}$ measured with the K\&ZLAS09 and the WagLAS. $C_{n}^{2}$ was evaluated in three different ways. Firstly, $C_{n}^{2}$ derived from the demodulated signal sampled with an integration time of $40 \mu \mathrm{s}$. Secondly, $C_{n}^{2}$ derived from the $V_{C_{n}^{2}}$ signal sampled with an integration time of $250 \mu \mathrm{s}$. Thirdly, $C_{n}^{2}$ derived from the demodulated signal sampled with an integration time of $250 \mu \mathrm{s}$

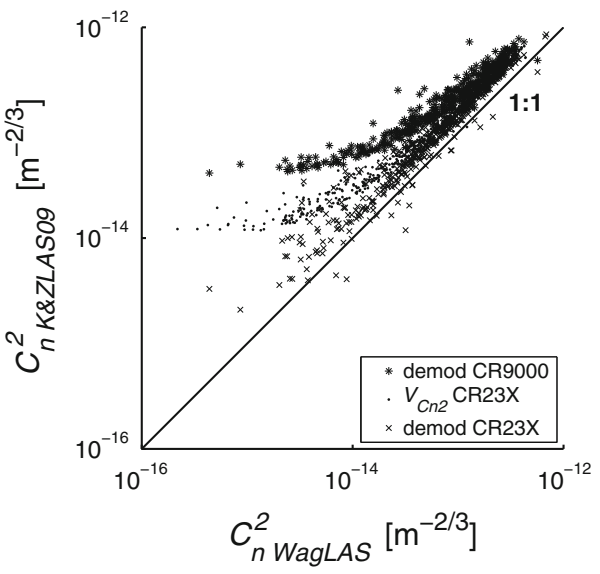


Zonen recommends users to store the variance of the demodulated signal, most users can evaluate $C_{n}^{2}$ in this way. Thirdly, $C_{n}^{2}$ is derived from the demodulated signal sampled on the CR9000 with a frequency of $500 \mathrm{~Hz}$ and an integration time of $40 \mu \mathrm{s}$. These are more specialist measurements and most users cannot evaluate $C_{n}^{2}$ in this way.

Figure 7 shows that the low- $C_{n}^{2}$ error depends on the way in which $C_{n}^{2}$ is evaluated. The low- $C_{n}^{2}$ error is the largest for $C_{n}^{2}$ derived from the demodulated signal with $40 \mu$ s integration time, for $C_{n}^{2}$ derived from the $V_{C_{n}^{2}}$ signal the error is smaller, whereas for $C_{n}^{2}$ derived from the demodulated signal with $250 \mu \mathrm{s}$ integration time the error has been reduced to zero. As can be seen, a key aspect in this is the signal integration time of the data logger. Looking at the $C_{n}^{2}$ estimates derived from the demodulated signal we observe that increasing the integration time removes the low- $C_{n}^{2}$ error.

However, for $C_{n}^{2}$ derived from the $V_{C_{n}^{2}}$ signal this observation is only partly true. The reason for this is that there is noise on the demodulated signal when it is processed. Apparently the high-frequency noise is not completely filtered out by the filter in the Kipp \& Zonen LAS analogue electronics, or the noise is again introduced after that filter, which leads to an overestimated $V_{C_{n}^{2}}$ signal and thus $C_{n}^{2}$.

Figure 7 also shows that the spectral peak associated with the low- $C_{n}^{2}$ error is aliased from higher frequencies. It would show up as a $100 \mathrm{~Hz}$ peak in the frequency spectrum derived from measurements with a $40-\mu$ s integration time, but it is averaged out by an integration time of $250 \mu \mathrm{s}$. Hence we conclude that the low- $C_{n}^{2}$ error is caused by noise with a frequency that is greater than $4 \mathrm{kHz}(250 \mu \mathrm{s})$. Together with the points discussed above, and considering that the main difference between the K\&ZLAS and WagLAS electronics is the inclusion of a calibration unit in the K\&ZLAS receiver, leads us to the conclusion that the calibration unit is the most likely noise source.

As a solution for the problem of the low- $C_{n}^{2}$ error we recommend users to evaluate $C_{n}^{2}$ derived from the variance and average of the demodulated signal, using Eqs. 3 and 1. Note that in doing so, we ignore the band-pass filtering included in the $C_{n}^{2}$ derived from $V_{C_{n}^{2}}$. For path lengths greater than $500 \mathrm{~m}$ the effect of ignoring this filter is limited, especially when absorption fluctuations are small. As a result, and depending on the humidity, our solution will still somewhat overestimate $C_{n}^{2}$, but this is only a fraction compared to the effect of the low- $C_{n}^{2}$ error, which is corrected for by this procedure.

To check the quality of $C_{n}^{2}$ thus obtained one can compare with $C_{n}^{2}$ derived from the $V_{C_{n}^{2}}$ signal. Figure 8 shows this comparison and it can be observed that the WagLAS is indeed free of the low- $C_{n}^{2}$ error, whereas the K\&ZLAS09 is not free of this error. Furthermore, in this way one can also identify whether the calibration of the potentiometer setting to set the path length is still valid or if the potentiometer was set to an incorrect value. If not valid or incorrect, this would show that the scatter in Fig. 8 would have a constant offset from the 1:1 line. Of course also a reference scintillometer can be used to validate these results.

The magnitude of the low- $C_{n}^{2}$ error is different for each K\&ZLAS. Its effect on the resulting heat flux for neutral to near-neutral conditions is:

$$
\frac{H \pm d H}{H} \propto\left(\frac{C_{n}^{2} \pm d C_{n}^{2}}{C_{n}^{2}}\right)^{0.5},
$$

where $d H$ and $d C_{n}^{2}$ are the errors in $H$ and $C_{n}^{2}$ respectively. Of all K\&ZLASs the K\&ZLAS09 suffers most from the low- $C_{n}^{2}$ error. For this scintillometer, with $C_{n}^{2}$ derived from the $V_{C_{n}^{2}}$ signal, the error is typically $\approx 20 \mathrm{~W} \mathrm{~m}^{-2}$ for $H<10 \mathrm{~W} \mathrm{~m}^{-2} \approx 5 \mathrm{~W} \mathrm{~m}^{-2}$ for $H \approx 50 \mathrm{~W} \mathrm{~m}^{-2}$, and it is negligible for $H>100 \mathrm{~W} \mathrm{~m}^{-2}$. Hence, for this instrument the low- $C_{n}^{2}$ error is significant for $H \leq 50 \mathrm{~W} \mathrm{~m}^{-2}$. 

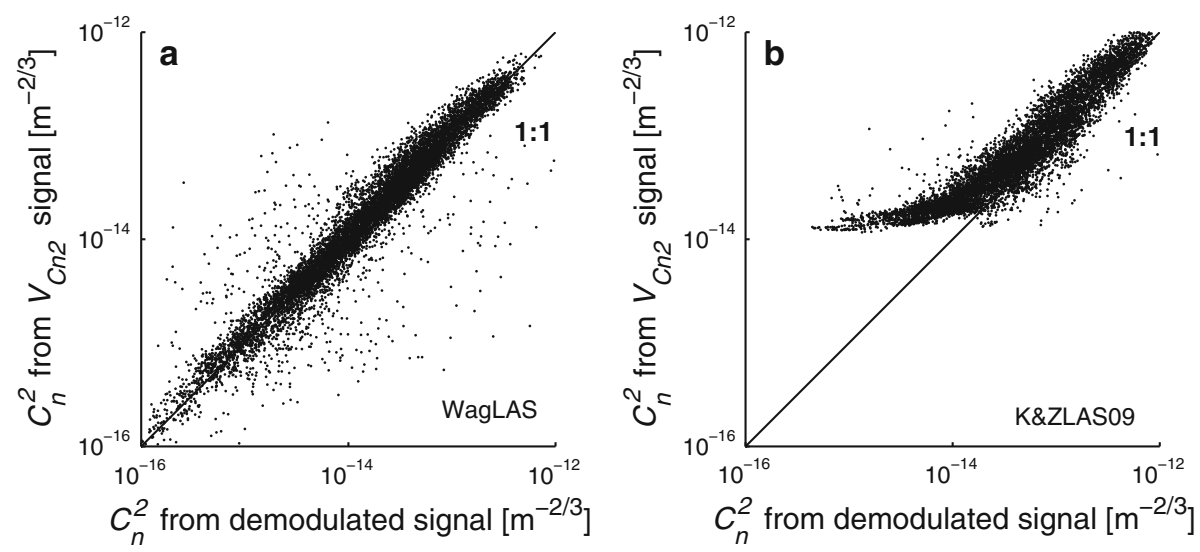

Fig. 8 LAS signal comparison of $C_{n}^{2}$, from the demodulated signal sampled with an integration time of $250 \mu \mathrm{s}$ and the $V_{C_{n}^{2}}$ signal, a for the WagLAS and $\mathbf{b}$ the K\&ZLAS09. Each data point is derived from 1-min statistics, and only limited data filtering was done for them

Table 2 Linear regression coefficients of the K\&ZLASs

\begin{tabular}{llllll}
\hline Scintillometer & $r$ & $a$ & $b\left(\mathrm{~m}^{-2 / 3}\right)$ & $n$ & $D_{\text {eff }}(\mathrm{m})$ \\
\hline K\&ZLAS09 & 0.998 & $1.53 x$ & $1.3 \times 10^{-14}$ & 549 & 0.127 \\
K\&ZLAS01 & 0.999 & $1.35 x$ & $8.8 \times 10^{-15}$ & 115 & 0.134 \\
K\&ZLAS30 & 0.990 & $3.40 x$ & $3.4 \times 10^{-15}$ & 307 & 0.090 \\
K\&ZLAS29 & 0.996 & $3.12 x$ & $1.4 \times 10^{-14}$ & 303 & 0.093 \\
\hline
\end{tabular}

$r$ is the correlation coefficient, $a$ is the regression slope, $b$ is the regression intercept, $n$ is the number of samples used and $D_{e f f}$ is the effective aperture diameter determined from Eq. 1 with $C_{n}^{2}$ from the WagLAS. All statistics are determined using a $95 \%$ confidence interval and derived from 30-min statistics

To place this into perspective: the uncertainty in estimating $H$ for neutral to near-neutral conditions is typically $10-20 \mathrm{~W} \mathrm{~m}^{-2}$ due to e.g. choice of MOST functions and estimated effective parameters as roughness length and effective height. Whether the low- $C_{n}^{2}$ error significantly contributes to the overall error in $H$ therefore depends on each K\&ZLAS instrument.

\subsubsection{Discussion and Solution of the High- $C_{n}^{2}$ Error}

In this section we continue with the high- $C_{n}^{2}$ error introduced in Sect. 4.2. To this end we present Table 2, where we show for $C_{n}^{2}$ derived from the $V_{C_{n}^{2}}$ signal the linear regressions parameters of all K\&ZLASs against the WagLAS. Note that, similar to Fig. 6, for Experiment $2 H_{W a g L A S}$ was reconstructed using the $H_{K \& Z L A S 09}$ versus $H_{W a g L A S}$ regression of Experiment 1.

Table 2 shows that all the scintillometers correlate very well with correlation coefficients of at least 0.99 . This confirms the results of Kleissl et al. $(2008,2009)$ and shows the high quality of the scintillometer measurements. The high- $C_{n}^{2}$ error, however, is large: the Kipp $\&$ Zonen LASs overestimate $C_{n}^{2}$ by at least $35 \%$ and up to $240 \%$. 
The magnitude of the high- $C_{n}^{2}$ error is different for each Kipp \& Zonen LAS. Its effect on the resulting heat flux under free convection conditions is:

$$
\frac{H \pm d H}{H} \propto\left(\frac{C_{n}^{2} \pm d C_{n}^{2}}{C_{n}^{2}}\right)^{0.75}
$$

Since the offsets, $b$, in the regression equations are small we can assume that the multiplication factor, $a$, is a good approximation to the high- $C_{n}^{2}$ error. For the K\&ZLAS09 this would suppose a relative error of $53 \%$ in $C_{n}^{2}(a=1.53)$ and consequently a relative error of $37 \%$ in $H$. To show that this is a fair approximation we take the regression equations from Fig. 3. For $H_{E C}=250 \mathrm{~W} \mathrm{~m}^{-2}$ we find that the relative error in $H$ is $\approx 33 \%$. One has to realize that the accuracy of Eqs. 5 and 6 is determined by the validity of the neutral or the free-convection assumption on which they are based. The true error, however, is always between these two limits.

One suspect for the high- $C_{n}^{2}$ error is the optical quality of the Fresnel lenses in the K\&ZLASs. The quality of these is known to be less than that of the concave mirror used in the WagLAS. As a result the effective diameter, $D_{e f f}$, seems to be smaller than the geometrical diameter of $0.152 \mathrm{~m}$. From Eq. 1 it follows that overestimating $D$ leads to overestimating $C_{n}^{2}$. This is consistent with what we observe.

However, Kipp \& Zonen did several tests to determine $D_{\text {eff }}$ by measuring the mean demodulated signal using a range of aperture sizes. They determined $D_{\text {eff }}$ by extrapolating the linear relationship between the aperture diameter and the square root of the mean demodulated signal. Kipp \& Zonen found typical values for $D_{\text {eff }}$ that were smaller than the physical lens diameter but were larger than $0.14 \mathrm{~m}$ (Martin Veenstra, Kipp \& Zonen, personal communication 2010; Kleissl et al. 2008). We also conducted this test using a K\&ZLAS instrument that was not part of the Chilbolton experiment and found $D_{\text {eff }}$ to be $0.141 \pm 0.001 \mathrm{~m}$.

To test whether these values of $D_{\text {eff }}$ explain the observed difference in $H$ we apply an alternative approach to determining $D_{e f f}$, and derive this from the comparison between the K\&ZLAS and our reference scintillometer, the WagLAS. From Eq. $1 D_{\text {eff }}$ of the K\&ZLAS can be determined using $C_{n}^{2}$ from the WagLAS, and $\sigma_{\ln I}^{2}$ and $L$ from the K\&ZLAS. $D_{\text {eff }}$ thus determined is listed in Table 2. It can be seen that the largest $D_{\text {eff }}$ is $0.134 \mathrm{~m}$, which is smaller than the typical values found by Kipp \& Zonen and ourselves in the test with the smaller apertures. Moreover, the values of $0.093 \mathrm{~m}$ and $0.090 \mathrm{~m}$ are so low that it is very unlikely a problem of the Fresnel-lens quality only.

Another contribution to the high- $C_{n}^{2}$ error could be poor alignment of the detector in the focal point of the lens. Recall that Kleissl et al. (2008) reported that the effect of a misaligned photodiode detector resulted in a $35 \%$ error in $H$; poor alignment causes the detector to be lit by a part of the lens only. Two design issues enhance the K\&ZLAS sensitivity to poor detector alignment: firstly, the fact that the detector is mounted on a large metal block (Kipp \& Zonen refer to this block as the bullet (Kipp and Zonen 2007)), which because of its bulkiness makes accurate alignment difficult. Secondly, the focal length of the Fresnel lens is $0.152 \mathrm{~m}$ whereas the WagLAS mirror has a focal length of $0.30 \mathrm{~m}$ (Kipp and Zonen 2007; Meijninger 2003). This implies that for the K\&ZLAS instrument a displacement away from the focal point in the lens-detector plane has a larger impact on the alignment than a similar displacement has on the alignment of the WagLAS. Poor focal-point alignment affects both the transmitter and the receiver. We did not test which of the two has a stronger influence on the high- $C_{n}^{2}$ error. Kipp \& Zonen found $D_{\text {eff }}$ to be $0.148 \mathrm{~m}$ for the receiver and 0.145 for the transmitter (Kipp and Zonen 2007). 
The high- $C_{n}^{2}$ is a linear error and consequently affects the whole range of $H$. We cannot offer a solution for the high- $C_{n}^{2}$ error other than checking the focal-point alignment or, if this cannot be done with sufficient accuracy, comparing the K\&ZLAS in the field against a reference scintillometer. The fact that the four K\&ZLASs all have a different high- $C_{n}^{2}$ error, and that their corresponding $D_{\text {eff }}$ is lower than can be expected from Fresnel-lens quality, supports our hypothesis that the main cause for this error is the focal-point alignment of the detector.

\section{Conclusions}

Kleissl et al. $(2008,2009)$ showed that there is a systematic error in the K\&ZLAS scintillometer measurements of $H$. Although these studies clearly allowed this general conclusion, the measurement conditions were not ideal, nor did they study the characteristics of the systematic error.

We conducted two experiments at the Chilbolton Observatory, where we compared four K\&ZLASs with a reference scintillometer, the WagLAS. This experiment was conducted under highly controlled conditions and we analyzed the characteristics of the error. We improved on the Kleissl experiments by installing all scintillometers close together (lateral spacing of $1.5 \mathrm{~m}$ in Experiment 1 and $7 \mathrm{~m}$ in Experiment 2) at exactly the same height, and over a path of $500 \mathrm{~m}$ length. This ensured the footprints to be the same, making a good comparison possible. To validate the scintillometer measurements an eddy-covariance system measured the sensible heat flux, $H$, halfway along the path at approximately the same height as the scintillometers. In addition, the scintillometers were placed in special research cabins on tables that stood independently from the cabins on large concrete pillars to avoid instrument-movement induced noise on the scintillometer signals.

Furthermore high-frequency sampling was done to enable spectral analysis of the data. From our comparison between the four K\&ZLASs with the WagLAS we draw two main conclusions. Firstly, our study shows that the scatter between the scintillometers is very low with correlation coefficients $\geq 0.99$. This confirms the results of previous studies, shows the robustness of the scintillometer method, and that the large-aperture scintillometer outperforms any other measurement system that obtains $H$. Secondly, unlike the Kleissl studies (2008, 2009), we found two systematic errors in the K\&ZLAS measurements, namely a high- $C_{n}^{2}$ error and a low- $C_{n}^{2}$ error that both result in an overestimation of $C_{n}^{2}$ and thus $H$.

The low- $C_{n}^{2}$ error is a non-linear error that is significant for $H \leq 50 \mathrm{~W} \mathrm{~m}^{-2}$ and varies significantly between each scintillometer. Typical values for the K\&ZLAS09, which suffers most from this error, are $\approx 20 \mathrm{~W} \mathrm{~m}^{-2}$ for $H<10 \mathrm{~W} \mathrm{~m}^{-2}$. The low- $C_{n}^{2}$ error has its origin in the receiver and was detected by spectral analysis of the demodulated raw signal. The error shows up as a peak in the spectrum at frequencies of $100 \mathrm{~Hz}$ or higher. We suspect that the calibration circuit within the receiver is the source of this error. We showed that the low- $C_{n}^{2}$ error does affect the $V_{C_{n}^{2}}$ signal, which is the output most users use. To remove this error we provided a procedure that estimates $C_{n}^{2}$ using the variance of the demodulated signal.

The high- $C_{n}^{2}$ error is a linear error that also varies significantly between each K\&ZLAS (overestimation from $35 \%$ up to $240 \%$ ), and it is the same error as that was observed by Kleissl et al. $(2008,2009)$. A high- $C_{n}^{2}$ error of $53 \%$ in the K\&ZLAS09 measurements of $C_{n}^{2}$ resulted in a 33\% error in $H$. As this is a linear error it affects the whole range of $H$. We suspect poor focal-point alignment of the receiver detector and the transmitter light-emitting diode, which leads to ineffective use of the Fresnel lens, to be the cause. Unfortunately there 
is no correction for the high- $C_{n}^{2}$ error other than to calibrate the K\&ZLAS against a reference scintillometer in the field.

Acknowledgements First of all we thank Charles Wrench and Darcy Ladd from the Chilbolton Observatory for making their wonderful facilities available to us. Furthermore we thank Jonathan Evans from the Centre for Ecology and Hydrology, Wallingford for his great help during the experiment and providing two K\&ZLASs to us. We also thank Sue Grimmond and Mariana Gouvea from King's College London for their help and making available to us two K\&ZLASs. Furthermore, we thank Jan, Mal, Judy, Joe, Owain, Dave, and Lorna for their assistance, kindness and hospitality during all parts of the experiment. Finally we thank two anonymous reviewers for their valuable comments. This research was supported by the Dutch Technology Foundation (STW) under project number WTC7484.

Open Access This article is distributed under the terms of the Creative Commons Attribution Noncommercial License which permits any noncommercial use, distribution, and reproduction in any medium, provided the original author(s) and source are credited.

\section{References}

Andreas EL (1988) Estimating $C_{n}^{2}$ over snow and sea ice from meteorological data 2. J Opt Soc Am 5:481-495

Andreas EL (1989) Two-wavelength method of measuring path-averaged turbulent surface heat fluxes. J Atmos Ocean Technol 6:280-292

Asanuma J, Iemoto K (2007) Measurements of regional sensible heat flux over Mongolian grassland using large aperture scintillometer. J Hydrol 333:58-67

Beyrich F, De Bruin HAR, Meijninger WML, Schipper JW, Lohse H (2002) Results from one-year continuous operation of a large aperture scintillometer over a heterogeneous land surface. Boundary-Layer Meteorol 105:85-97

De Bruin HAR, Van den Hurk B, Kohsiek W (1995) The scintillation method tested over a dry vineyard area. Boundary-Layer Meteorol 76:25-40

Green AE (2001) The practical application of scintillometers in determining the surface fluxes of heat, moisture and momentum. PhD Thesis, Wageningen University, Wageningen, The Netherlands, $177 \mathrm{pp}$

Hartogensis OK (2007) BSIK-ME2 scintillometer network. http://www.met.wau.nl/index.html; http://www. met.wau.nl/projects/BSIK_ME2/BSIK_index.html. Accessed 19 March 2007

Hartogensis OK, Watts CJ, Rodriquez J-C, De Bruin HAR (2003) Derivation of an effective height for scintillometers: La Poza experiment in northwest Mexico. J Hydrometeorol 4:915-928

Kaimal JC, Finnigan JJ (1994) Atmospheric boundary layer flows-their structure and measurement. Oxford University Press, New York, 289 pp

Kipp \& Zonen (2007) Instruction manual large aperture scintillometer, version 0307. Kipp \& Zonen B.V. Delft, The Netherlands, $73 \mathrm{pp}$

Kleissl J, Gomez J, Hong SH, Hendrickx JMH, Rahn T, Defoor WL (2008) Large aperture scintillometer intercomparison study. Boundary-Layer Meteorol 128:133-150

Kleissl J, Watts CJ, Rodriguez JC, Naif S, Vivoni ER (2009) Scintillometer intercomparison study-continued. Boundary-Layer Meteorol 130:437-443

Meijninger WML (2003) Surface fluxes over natural landscapes using scintillometry. PhD Thesis, Wageningen University, Wageningen, The Netherlands, $164 \mathrm{pp}$

Meijninger WML, De Bruin HAR (2000) The sensible heat fluxes over irrigated areas in western Turkey determined with a large aperture scintillometer. J Hydrol 229:42-49

Meijninger WML, Hartogensis OK, Kohsiek W, Hoedjes JCB, Zuurbier RM, De Bruin HAR (2002) Determination of area-averaged sensible heat fluxes with a large aperture scintillometer over a heterogeneous surface-Flevoland field experiment. Boundary-Layer Meteorol 105:37-62

Meijninger WML, Beyrich F, Luedi A, Kohsiek W, De Bruin HAR (2006) Scintillometer-based turbulent fluxes of sensible and latent heat over a heterogeneous land surface-a contribution to LITFASS-2003. Boundary-Layer Meteorol 121:89-110

Moene AF (2003) Effects of water vapour on the structure parameter of the refractive index for near-infrared radiation. Boundary-Layer Meteorol 107:635-653

Moene AF, Meijninger WML, Hartogensis OK, Kohsiek W, De Bruin HAR (2004) A review of the relationships describing the signal of a large aperture scintillometer. Internal Report 2004/2, Meteorology and Air Quality Group, Wageningen University, Wageningen, the Netherlands, 39 pp 
Moore CJ (1986) Frequency response corrections for eddy correlation systems. Boundary-Layer Meteorol 37:17-35

Nieveen JP, Green AE, Kohsiek W (1998) Using a large-aperture scintillometer to measure absorption and refractive index fluctuations. Boundary-Layer Meteorol 87:101-116

Ochs GR, Wang T (1978) Finite aperture optical scintillometer for profiling wind and $C_{n}^{2}$. Appl Opt 17:37743778

Oncley S (2007) Combing short-term moments for longer time periods. NCAR, Atmosphere Technology Division (ATD), Integrated Surface Flux Facility. http://www.eol.ucar.edu/instrumentation/sounding/ isfs/isff-support-center/how-tos/combining-short-term-moments-for-longer-time-periods. Accessed 28 September 2010

Schotanus P, Nieuwstadt FTM, De Bruin HAR (1983) Temperature measurement with a sonic anemometer and its application to heat and moisture fluxes. Boundary-Layer Meteorol 26(1):81-93

Van Dijk A, Moene AF, De Bruin HAR (2004) The principles of surface flux physics: theory, practice and description of the ECPACK library. Internal Report 2004/1, Meteorology and Air Quality Group, Wageningen University, Wageningen, the Netherlands, $99 \mathrm{pp}$

Van Kesteren B (2008) Sensible and latent heat fluxes with optical and millimetre wave scintillometers. Master Thesis, Wageningen University, Wageningen, the Netherlands, 99 pp

Wang TI, Ochs GR, Clifford SF (1978) Saturation-resistant optical scintillometer to measure $C_{n}^{2}$. J Opt Soc Am 68(3):334-338

Wrench CL (2003-2010) Chilbolton Facility for Atmospheric and Radio Research (CFARR) data. Science and Technology Facilities Council (STFC), Chilbolton Facility for Atmospheric and Radio Research. http://badc.nerc.ac.uk/data/chilbolton/. Accessed 25 September 2007 